The centering error of a sextant

This content has been downloaded from IOPscience. Please scroll down to see the full text. 1918 Trans. Opt. Soc. 201

(http://iopscience.iop.org/1475-4878/20/1/302)

View the table of contents for this issue, or go to the journal homepage for more

Download details:

IP Address: 143.167.2.135

This content was downloaded on 08/09/2015 at 00:52

Please note that terms and conditions apply. 


\section{TRANSACTIONS OF THE}

OPTICAL SOCIETY

Vol. XX.

OCTOBER, I9I8

No. I.

\section{THE CENTERING ERROR OF A SEXTANT}

By Naval Instructor T. Y. BAKER, R.N.

Read and discussed, roth October, 1918

THE accurate performance of a sextant depends among other things upon the centre round which the index arm turns being coincident with the centre from which the graduations are cut. If there is a separation between these two points an error in the reading will result whose value is easily determinable if the displacement of the one centre relative to the other is known.

In Fig. I let $A$ be the mechanical centre and $B$ the centre of the scale. Let $P$ be the position of the index datum mark when measuring an angle $\theta$. Let the line of centres $B A$ produced cut circles of the same radius but with centres at $B$ and $A$ in $B^{\prime}$ and $A^{\prime}$ respectively.

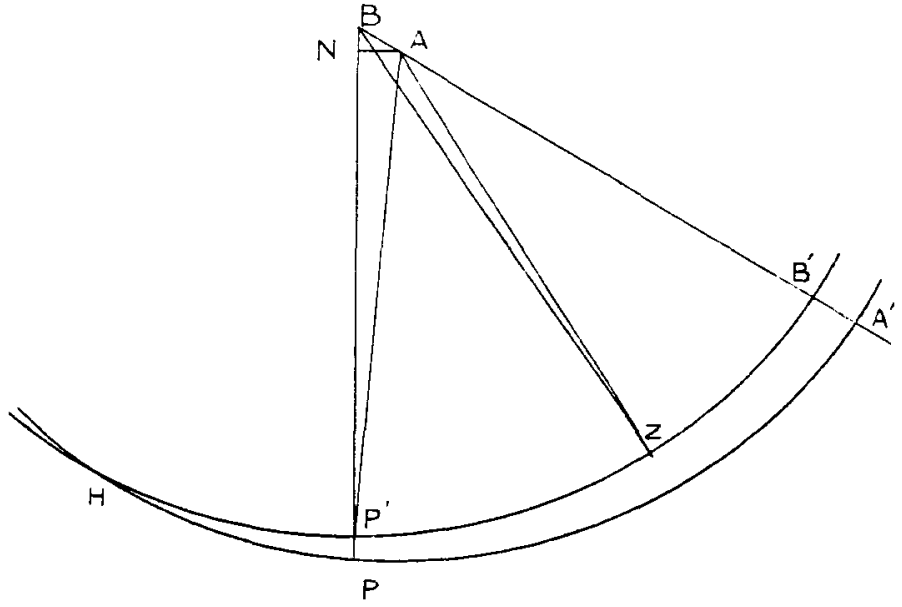

Fig. I 
Now the actual measurement of an angle is made by doubling the angular rotation of the movable mirror from the position at which the two mirrors are parallel to one another. This position does not in general coincide with the zero of the scale and a consequent zero or index error has to be applied to the reading. Such a zero error is constant all along the scale and it is immaterial whether it is large or small provided it can be determined. The centering error on the other hand varies from point to point along the arc. It will be convenient therefore for the purpose of a preliminary consideration of the centering error if we imagine that the zero of the scale starts at $B^{\prime}$. There will then be no centering error at that position as the two centres are in line. When the index arm has moved to $P$ it will have turned through an angle $P A A^{\prime}$ but the angle subtended at the centre of the scale is $P B A^{\prime}$.

The angular value of the centering error is $B P A$ which has really to be doubled when we speak of it as a correction to the reading.

If $r$ is the radius of the index arm the value of the centering error is easily seen to be (in circular measure) $2 A N / A P$ or $2 A B \sin A B P / r$.

Now let $Z$ be the true zero of the scale, i.e. $A Z$ represents the position of the index arm when the two mirrors are parallel. Then the value of the centering error at $P$ may be expressed as

$$
2 A B \sin \left(\frac{\theta}{2}+Z A B^{\prime}\right) / r
$$

If $\epsilon$ is the angular excentricity of the centre $B$ (i.e. $A B / r$ ) and $Z A B^{\prime}={ }_{2}^{\alpha}$ we can write

$$
\text { Centering error }=2 \epsilon \sin \frac{1}{2}(\theta+\alpha) \text {. }
$$

When plotted on a base of readings we obtain the following type of curve.

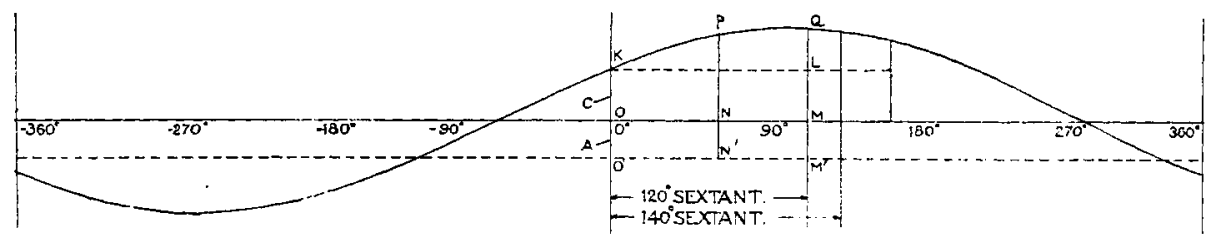

Fig. 2

This is a curve of sines starting at a position $-\alpha$ (which may be anywhere between $0^{\circ}$ and $720^{\circ}$ ) and having a maximum height of $2 \varepsilon$.

Now even if there is no centering error there can still be a zero error through the scale starting at the wrong point. Therefore in determining the error of the reading $(R)$ we must write it in the form

$$
\partial R=A+2 \epsilon \sin \frac{1}{2}(\theta+d),
$$

or, more generally, the reading error is of the form

$$
\partial R=A+B \sin \frac{1}{2} \theta+C \cos \frac{1}{2} \theta,
$$

where $A, B$ and $C$ are constants whose values depend upon the starting point of the scale and the magnitude and direction of the displacement of the centre. 
Fig. I clearly shews that there is a centering error at the zero position, viz. $2 B Z A$; the value of this is

$$
\partial R_{0}=A+C \text { by putting } \theta=0 .
$$

In Fig. 2 the dotted line is drawn parallel to the base of the curve at a distance $A$ below it. Ordinates of the curve therefore measured from the level of the dotted line are the corrections that have to be applied to the reading, for example $P N N^{\prime}$ is the correction for a reading $O N$. Now the range of the sextant is usually not more than $140^{\circ}$ and very frequently only $120^{\circ}$. For convenience in calculation we will adopt the latter figure and replace it when needed by the higher one. The direction of the excentricity may be anything so that the starting point of the curve may be anywhere (in Fig. 2) between $=360^{\circ}$. This is the same as saying that the reading error of the sextant is shewn as a graph by cutting out a proper portion (which may be any portion) of $120^{\circ}$ base from the curve shewn in Fig. 2. With the figure as drawn the error will vary between $K O^{\prime}$ and $Q M^{\prime}$ where $Q M^{\prime}$ is drawn at $120^{\circ}$.

Now $K O^{\prime}$ is really the angle which is measured when determining the zero error. It is the reading of the sextant when the two mirrors are set parallel to one another. Hence the variable part of the centering error is the ordinate of the curve $K Q$ measured from the level $K L$ and it is now clear how the direction of the displacement will affect the value of the centering error over the range of the readings.

The best case, given a definite value for $A B$ in Fig. I, is for the displacement of the centre to be at right angles to the radius to the middle of the arc; the curve will then be placed as shewn in Fig. 3 .

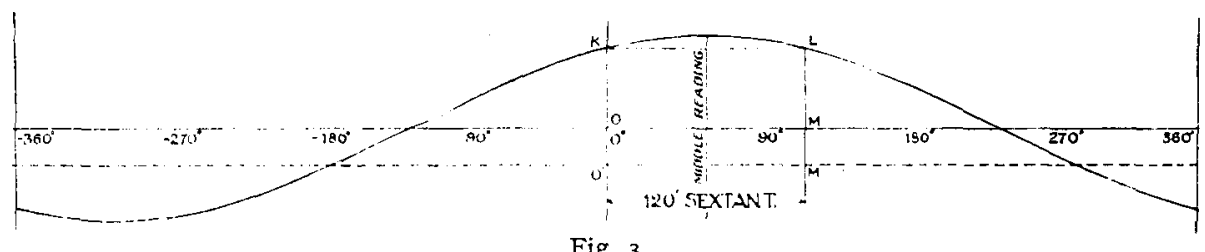

Fig. 3

The variable part of the centering error is then zero at $0^{\circ}$ and $120^{\circ}$ and runs to a maximum of $2 \epsilon\left(1-\sin 60^{\circ}\right)$, or $0.268 \epsilon$, at the middle reading.

If we take a sextant reading up to $I+0^{\circ}$ the corresponding formula is

$$
2 \epsilon\left(1-\sin 55^{\circ}\right) \cdots 0.362 \epsilon \text {. }
$$

This is not quite true. By shifting the base line a little further to the right the centering error may be lessened by making it go to a maximum positive value before the middle reading and become zero again before the end reading. For the remainder of the scale the error will be negative.

The centering error as determined by the N.P.L. will therefore be between the two values $0.268 \epsilon$ and $0.362 \epsilon$ provided that the centre is out in the best direction, i.e. at right angles to the middle reading. To obtain an " $A$ " certificate this error must not exceed $40^{\prime \prime}$ and under such circumstances the value of $\epsilon$ may be as much as $150^{\prime \prime}$ for the $120^{\circ}$ sextant and I $10^{\prime \prime}$ for the other. With a seven inch radius this corresponds to an excentricity of 5 mils in the first case and 4 mils in the second. 
These are the largest amounts of displacements that are allowable for a seven inch sextant with an "A" certificate and it is only possible to permit of these provided the centre is displaced at right angles to the middle reading.

The other extreme, the worst possible case, will be that for which the middle reading corresponds to the starting point of the curve of sines. The curve in this case is shewn in Fig. 4.

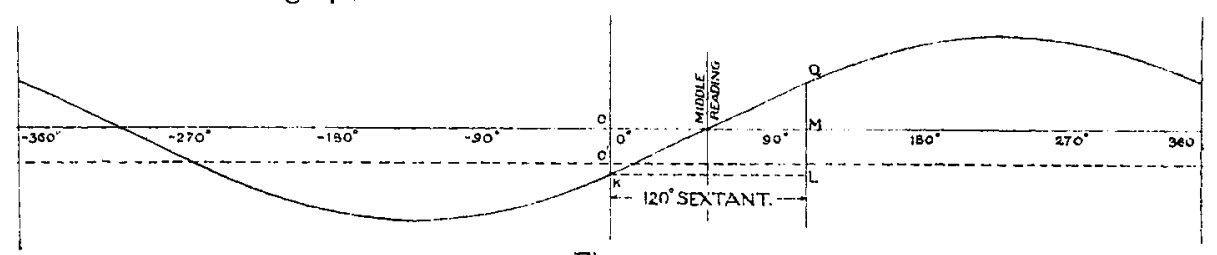

Fig. 4

The greatest amount of the centering error is $Q L$ at the top reading which is seen from the figure to be equal to $4 \epsilon \sin 30^{\circ}$ in the case of the sextant reading up to $120^{\circ}$ and $4 \epsilon \sin 35^{\circ}$ in the case of the sextant reading up to $140^{\circ}$.

These amounts are $2 \epsilon$ and $2 \cdot 294 \epsilon$ and permit of a displacement of the centre of a seven inch sextant of $0.7 \mathrm{mil}$ and $0.6 \mathrm{mil}$ respectively for sextants reading up to $120^{\circ}$ and to $140^{\circ}$. These investigations cover the maximum possible displacements of the centre according to the direction in which that displacement takes place, viz. at right angles to, and along, the line of the middle reading.

For any other direction of displacement the amount of displacement is intermediate between these two and the area within which the centre must lie is a tankshaped area as shewn in Fig. 5 .

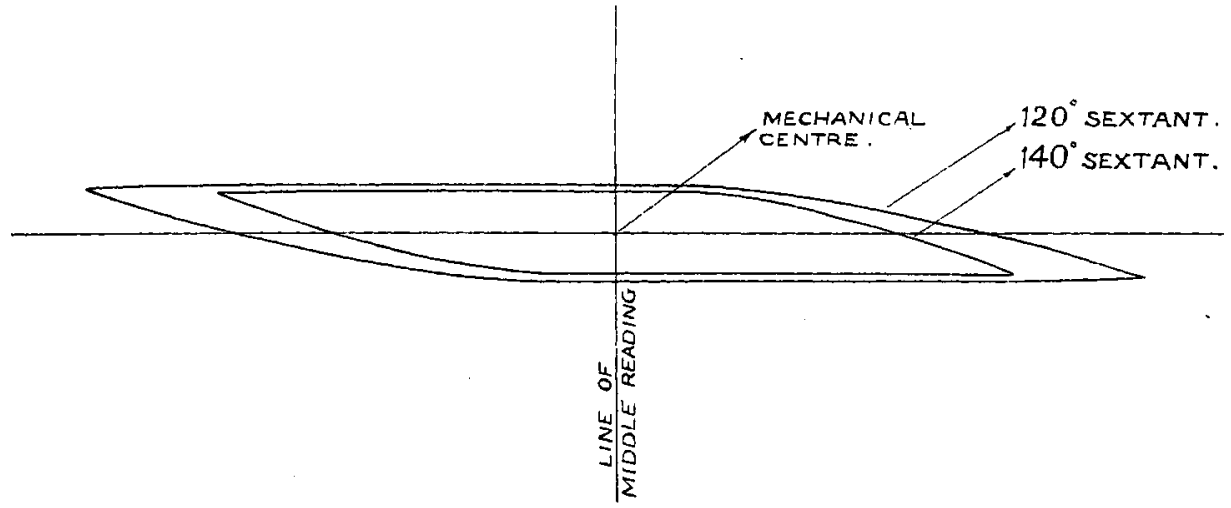

Fig. 5

It will be seen from this figure, which is drawn to scale, that the 5 mils excentricity for a displacement at right angles to the middle reading is not quite the maximum, but that the latter is found along a slightly different direction, viz. to the nose or tail of the tank.

We shall see later that in view of the vernier reading the amount of excentricity at right angles to the middle reading requires modification. 
Method of cutting the scale.

An ordinary method is to lay the limb of the sextant upon the turntable of the dividing engine and adjust it in such a manner that any excentricity is shewn by the long arm of a lever, the short arm of which is in contact with the side of the central hole, having no movement as the turntable is rotated. This arrangement of a lever ought to be such as to shew a total movement of the short arm equivalent to the short breadth of the area in Fig. 5, i.e. it must shew I. 4 mils with the short arm for a seven inch $120^{\circ}$ sextant, $\mathrm{I} \cdot 2$ mils for a seven inch $140^{\circ}$ instiument.

A mechanical magnification of this is hardly possible above five times, in which case the long arm must shew a movement of 7 mils and 6 mils respectively.

All sextant makers know that this degree of accuracy is hardly ever obtained with the result that the sextant when it comes off the dividing engine has in the vast majority of cases a centering error which is too big for the " $\mathrm{A}$ " certificate and the mechanical centre is usually taken out and re-adjusted as near as possible to coincide with the scale centre.

\section{Means of re-adjustment of centre.}

The maker of course wishes to know before sending the sextant for its official test, how nearly correct the centre is, and the workshop methods of testing for this

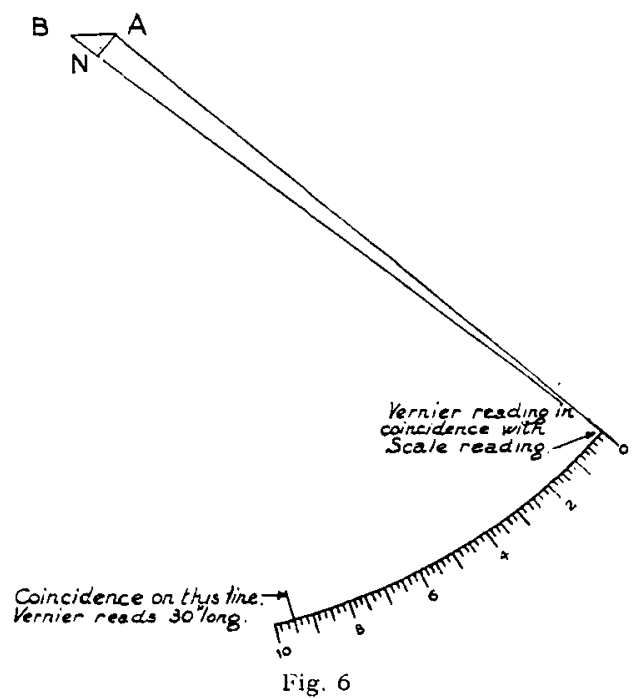

scem to leave much to be desired. The customary practice is to compare the end vernier readings at different places along the arc. If the vernier reads long, i.e. the vernier distance is greater than the scale distance, it is a sign that the vernier is too near the scale centre.

Generally speaking 60 divisions of the vernier correspond with 119 of the scale and most stxtant users can be sure of a reading to one division but not much closer 
than that. A skilled man will probably do better but it is very much doubted whether the most highly trained sextant adjuster is able to be certain whether 60 divisions of the vernier are in coincidence with marks on the arc or whether when one end is exact the other end is half a division long or short.

Putting the precision at Io sextant seconds the value of this method of determining the centering accuracy is easily determinable. When the excentricity is at right angles to the index arm the method gives no indication of the fact; when the excentricity is in the direction of the index arm the method is working under the best possible conditions. The length of the vernier is normally i i 9 scale divisions, i.e. $19^{\circ} 50^{\prime}$ (sextant angle). If the vernier reads $10^{\prime \prime}$ too long the radius is too short by $\frac{\mathrm{rO}^{\prime \prime}}{19^{\circ} 50^{\prime}}$ of its length. For a 7 inch sextant this equals $\mathrm{I}$ mil excentricity.

The radius of the index arm may, however, in itself be different from that of the scale so that it is the variation of the vernier length which is what the adjuster has to deal with, and for a I mil excentricity the adjuster would have to be able to detect a difference in the vernier length equivalent to less than $\mathrm{I} \frac{1}{2}$ sextant seconds, i.e. a test which is a long way beyond his powers.

It has previously been stated that the centre of a 7 inch $120^{\circ}$ sextant may be excentric to the extent of 5 mils at right angles to the middle reading. With such a sextant no centering error would arise exceeding $40^{\prime \prime}$ provided that the zero of the vernier came exactly on a scale division. In such case, however, the vernier would be reading long or short and additional error would be introduced if the vernier zero did not lie exactly on a scale division, that is to say, if the sextant reading were not an even IO'. In Fig. 6 the excentricity $B A$ is given this maximum value. The vernier for zero reading is consequently $B A \cos 30^{\circ}$ too near the centre or 4.4 mils and the other end of the vernier would be $44^{\prime \prime}$ too long. The additional error will therefore be anything between $0^{\prime \prime}$ and $44^{\prime \prime}$ in the neighbourhood of the zero of the sextant and anything between $o^{\prime \prime}$ and $-44^{\prime \prime}$ in the neighbourhood of the other end of the arc, the amount depending upon the odd minutes and seconds of the reading above the even ro minutes.

The 5 mils excentricity that would be allowable if the reading were carried out by any other method than a vernier therefore requires modification. Probably the figure should be taken as not greater than 2 mils.

\section{An alternative method.}

Owing to this difficulty of testing the sextant in the workshop I put forward a proposal some years ago, which has since been adopted by the Admiralty and the N.P.L. in the specification for a Cadet's sextant, that while the instrument is fixed on to the dividing engine a circular arc should be engraved from the same centre as that from which the scale is cut. A mark is also made on the side of the index arm, as shewn in Fig. 7. The mark on the index arm has to come directly over the circular arc and in traversing the index arm from one end of the scale to the other the mark and the arc should track exactly. Any divergence from correct tracking will indicate that the mechanical and scale centres are not 
coincident. The tracking of this mark and circular arc needs very careful observation if the best uses are to be made of them, but an examination of the scheme appears to indicate that the correct adjustment of the centre is more possible by this means than by an examination of the vernier readings.

One of the great difficulties lies in the fact that when setting the vernier on the dividing engine for marking it will, in general, be put down excentrically and consequently the distance of the mark from the mechanical centre of the pivot

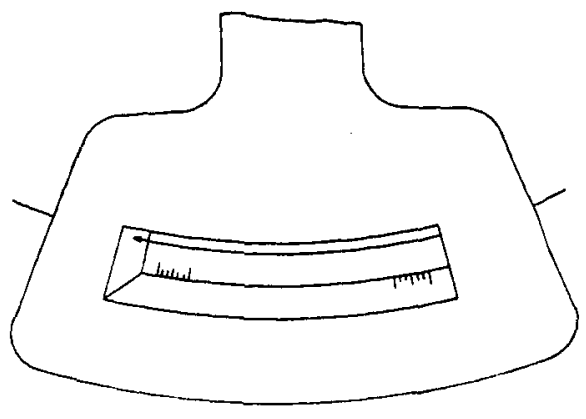

Fig. 7 will not be the radius with which the circular arc is cut on the limb. The amount of difference will depend upon the magnitude and direction of the displacement between the vernier scale centre and the mechanical centre of the index pivot.

The total amount of divergence therefore between the vernier mark and the circular arc of the scale includes the difference between the distance of the former from the mechanical centre and the radius of the latter. The mere fact that the vernier mark does not coincide with the circular arc is no test of correct centering of the scale; the proper test is as to whether the distance between the two varies as the vernier is moved from one end of the arc to the other.

We have seen that there are two extreme cases for the direction in which the scale centre may be excentric; the best case for a given amount of excentricity being that of an excentricity at right angles to the middle reading and the worst case an excentricity along the line of middle reading.

If the radii of the circular arc and of the vernier mark are exactly equal it follows from Fig. I that the amount of divergence between the two is $B N$ or $A B \cos A B N$. This divergence plotted on a base of readings would give an exactly similar curve to that shewn in Fig. 2 but $180^{\circ}$ (sextant angle) different in phase. Hence from whatever part of this curve we take the $120^{\circ}$ or $140^{\circ}$ that represents the centering error, a position $180^{\circ}$ away from it will be the corresponding curve of divergence.

In Fig. 3, where the centering error is zero at each end and a maximum at the middle reading, the divergence will be largest at each end and zero at the middle of the arc and it will obviously be easy to detect by the eye. On the other hand if the centering error rises to its maximum at the end of the arc as in Fig. 4 the divergence will be large all along the arc with a maximum value at the middle reading. But the fact that the divergence is large is also due possibly to the error in the radius to the vernier mark; it is the variation of the divergence that must be examined.

These two cases are considered in detail for both the $120^{\circ}$ and the $140^{\circ}$ sextant.

The first case corresponds to the excentricity being at right angles to the middle reading which allowed $\epsilon$ to be $15^{\prime \prime}$ (or 300 sextant seconds) with the $120^{\circ}$, and II $0^{\prime \prime}$ (or 220 sextant seconds) with the $140^{\circ}$ sextant. The total change in the divergence between the vernier mark and the circular arc is 300 sextant seconds 
and $25^{\circ}$ sextant seconds respectively. It is clear that such an excentricity is easily noticeable through the reading microscope as it involves a divergence of perhaps $15^{\prime \prime}$ on the one side of the mark to $15^{\circ}$ " on the other. These figures must be proportionately reduced in view of what has been stated concerning the necessity of cutting down the 5 mils to 2 mils. The other extreme case is unfortunately much more difficult to deal with. If the excentricity is along the line of middle reading then $\epsilon$ must not be greater than $20^{\prime \prime}\left(=40\right.$ sextant seconds) for the $120^{\circ}$ sextant, nor greater than $I 7^{\prime \prime}\left(=33\right.$ sextant seconds) for the $140^{\circ}$ sextant. The divergence now runs to a maximum at the middle reading and the change as compared with the end readings is 5 sextant seconds for the $120^{\circ}$ sextant and 6 sextant seconds for the $140^{\circ}$ sextant. As the change might be represented for the former case as

$$
\begin{array}{lccc}
\text { Reading } & 0^{\circ} & 60^{\circ} & 120^{\circ} \\
\text { Divergence } & -2 \cdot 5^{\prime \prime} & +2 \cdot 5^{\prime \prime} & -2 \cdot 5^{\prime \prime}
\end{array}
$$

it follows that this method applied to this case necessitates the adjuster being able to detect a divergence between the two lines equal to one-quarter of the $10^{\prime \prime}$ division of the vernier. This is of course putting it in the worst possible light. If the end divergence is zero the middle one will be $5^{\prime \prime}$ and if the end divergence is $5^{\prime \prime}$ the middle one will be $10^{\prime \prime}$. Things are slightly better for the $140^{\circ}$ sextant. The adjustment of the centre would therefore appear to be better carried out by means of the circular arc with the addition of observation of the length of the vernier than by the use of the latter method alone. Especially is this the case if a microscope with a micrometer eyepiece is used which can be fixed to the index arm of the sextant and the displacements of the circular arc examined as the index arm is turned round. A much higher magnification is then possible, the discrepancy between the radius of the circular arc and the radius to the vernier mark can be eliminated and possible errors in reading due to a very slight parallax between the vernier and the scale can be overcome. I suggest that if such a microscope is fitted the diaphragm of the focal plane of the eyepiece should carry not cross wires but two black circles separated by an amount which corresponds to about $20^{\prime}$ at the eye. These will be adjustable so that the circular arc bisects the space between them. The light which illuminates the scale must be rigidly attached to the microscope so that the circular arc is always illuminated from the same direction, preferably the direction of the portion of the arc being examined. I believe if this were done it would be possible to be sure by a careful adjustment of the centre that every sextant sent to be tested had no error exceeding $20^{\prime \prime}$ of arc due to centering error.

\section{Correct centering on the dividing Engine.}

We have examined the question of the centering error of a sextant under the assumption which is nearly always justified that the scale is not cut from the correct centre. Looking however at the whole problem of constructing accurate sextants it becomes a question whether this re-adjustment ought to be necessary. We have seen that to obtain an " $\mathrm{A}$ " certificate the scale centre must not be displaced more than 0.7 mil along the middle reading nor more than 2 mils at right angles to that line. It would seem that the greatest opening for improvement lies in the 
means of centering of the sextant on the dividing engine and that methods such as I have described should be scrapped in favour of some method of examining under a microscope the edge or the surface of the central hole while the table of the dividing engine is rotated. I do not desire to enter into this question too fully as different dividing engines are built in different ways and a method which is practicable with one would not be possible with another. But if I were a sextant maker and did my own dividing one of the first things I should turn my attention to would be the possibility of examining the concentricity of the sextant with the rotating table with such care that the limits of what $I$ have shewn is allowable should never be exceeded. The whole of the troublesome business of re-adjusting the centre would then be dispensed with.

\section{DISCUSSION}

Mr H. A. Hughes thought that the centering error was not one of the difficulties his firm experienced, judging from the present comparative infrequency of failure.

Dr J. S. Anderson said it would be interesting if $\mathrm{Mr}$ Baker would indicate what degree of accuracy was attained in measurements made under service conditions.

Mr Baker replied that with a 7 inch sextant measurements could be repeated to about $10^{\prime \prime}$ or $20^{\prime \prime}$ under good conditions. This accuracy was required in survey work, but not in sea work.

Mr H. S. Ryland pointed out that the sextant of to-day differs in mechanical design but little from that of thirty years ago. It is a mistake to attempt to locate the centre after dividing. Frequently, too, the arc is waved, due to distortion after dividing, and the correction of this error is in general of a temporary nature. Attention should be given to the illumination of the vernier, as errors amounting to $\pm .5^{\prime}$ may be produced if it is oblique.

Major Filon said that, as far as he knew, verniers were not used in observatory astronomical instruments such as transit circles, and he asked if it would not be possible to make use of micrometers instead of verniers on sextants.

$\mathrm{Mr} \mathrm{T}$. Smith thought the author had indicated the proper solution of eliminating centering errors, viz. the correct laying of the centres in coincidence on the dividing engine. If this were not possible it was advisable to adjust the centres approximately and then make the final adjustments by using both the vernier fit and the arc circle. If that were not sufficient. the final adjustment should be made by some optical means fitted on to the sextant.

With reference to Mr Hughes' remarks he would like to point out that a large proportion of sextant rejections were due to the corrections exceeding the limit allowed, i.e. to what makers usually call centering error whatever the real source may be.

Mr L. C. Martin drew attention to the question of the possible accuracy of reading. In the case of an instrument recently acquired by the Technical Optics Department differences of reading up to I minute could be obtained when side illumination was employed, owing to parallax and to differences in illumination of 
the scale and vernier. Some of the trouble could be eliminated by using a suitable magnifier with a telecentric stop at the focus. He thought that such a system had not been used in sextants.

He asked why the complete circle had not been employed in cases where weight was not of great importance.

$\mathrm{Mr} \mathrm{J}$. Guild stated that the question of the accumulative errors due to error in the vernier need not be considered, as in the N.P.L. "A" test the vernier length was tested at all parts of the scale, and the instrument rejected if the error in length anywhere exceeded 10 seconds with certainty. Further, a vernier would not be passed if there was appreciable parallax between it and the scale.

$\mathrm{Mr}$ S. D. Chalmers suggested the possibility of placing the scale on the dividing engine in such a way that any error should necessarily be in a definite direction.

The author, in reply, said the sextant should not have a removable centre; the scale should be laid down so carefully on the dividing engine as to ensure that the scale centre was not more than $1 / 10,000$ inch away from the mechanical centre.

$\mathrm{He}$ did not like the use of a vernier; something in the nature of a micrometer eyepiece would be better, even if it had to be doubled up so as to shorten its length. It was difficult to be sure that the light falls at equal angles on the vernier and scale, especially if the illumination was from the side.

The design of the centre was important. It should be broad and short rather than thin and long.

He did not agree with Mr Hughes' remarks; he thought that the usual cause of rejection was due to the centering error being greater than $40^{\prime \prime}$. 